\title{
A KATSYLO THEOREM FOR SHEETS OF SPHERICAL CONJUGACY CLASSES
}

\author{
GIOVANNA CARNOVALE AND FRANCESCO ESPOSITO
}

\begin{abstract}
We show that, for a sheet or a Lusztig stratum $S$ containing spherical conjugacy classes in a connected reductive algebraic group $G$ over an algebraically closed field in good characteristic, the orbit space $S / G$ is isomorphic to the quotient of an affine subvariety of $G$ modulo the action of a finite abelian 2-group. The affine subvariety is a closed subset of a Bruhat double coset and the abelian group is a finite subgroup of a maximal torus of $G$. We show that sheets of spherical conjugacy classes in a simple group are always smooth and we list which strata containing spherical classes are smooth.
\end{abstract}

\section{INTRODUCTION}

In 17, it is shown that the orbit space of a sheet $S$ of adjoint orbits in a complex Lie algebra has the structure of a geometric quotient which is isomorphic to an affine variety modulo the action of a finite group. The affine variety is the intersection of $S$ with the Slodowy slice of a nilpotent element $e$ in $S$, and the finite group is the component group of the centralizer of $e$. An algebraic proof of this result was obtained by Im Hof [16], who proved that sheets in complex Lie algebras of classical type are all smooth, by showing that $S$ is smoothly equivalent to its intersection with the Slodowy slice. Katsylo's theorem has also been applied to the study of one-dimensional representations of finite $W$-algebras [18, 23, which is related to the problem of determining the minimal dimensional modules for restricted Lie algebras. In this context, it has been shown in [24] that the space of 1-dimensional representations of the finite $W$-algebra associated with a nilpotent element $e$ in a classical Lie algebra is isomorphic to an affine space if and only if $e$ lies in a single sheet. The latter condition is equivalent to saying that the union of the sheets passing through $e$ is a smooth variety. Our goal is to provide an analogue of Katsylo's theorem for sheets of conjugacy classes in a reductive algebraic group $G$ over an algebraically closed field of good characteristic. Since sheets are the irreducible components of the parts in Lusztig's partition [20] called strata, the theorem will give an analogue for strata as well. As strata should be seen as the group analogue of the union of sheets passing through a nilpotent element $e$ (used in [24]), we expect that their geometry will have relevance in representation theory of quantum groups at the roots of unity. Evidence of this is visible in Sevostyanov's proof of the De Concini, Kac and Procesi conjecture on irreducible representations

Received by the editors January 19, 2015 and, in revised form, September 5, 2015 and September 10, 2015.

2010 Mathematics Subject Classification. Primary 20G15; Secondary 17B45.

The present work was partially supported by Progetto di Ateneo CPDA125818/12 of the University of Padova, FIRB 2012 Prospettive in Teoria di Lie and PRIN 2012 Spazi di Moduli e Teoria di Lie. 
of quantized enveloping algebras at the roots of unity [26. A conjugacy class in the corresponding simply-connected group is attached to each irreducible representation and the conjecture relates their dimensions. In the proof conjugacy classes lying in the same stratum are handled by using the same combinatorial data.

We prove a Katsylo theorem in the case that the sheet (or stratum) in question contains (hence consists of) spherical conjugacy classes, that is, classes having a dense orbit for a Borel subgroup $B$ of $G$. Strata, and therefore sheets, of conjugacy classes in a reductive algebraic group do not necessarily contain unipotent classes, so the analogue of Katsylo's theorem cannot be straightforward. A group analogue for Slodowy slices has been introduced in [25. In analogy to Steinberg's cross section, these slices depend on a conjugacy class in the Weyl group $W$ of $G$. The construction of these slices requires a suitable choice of positive roots in the root system of $G$ which depends on the class of the element in $W$. Although the transversality result in 25] is stated in characteristic zero, the proof holds in arbitrary characteristic. When $w \in W$ acts without fixed points, a section analogous to the one in [25] was given in [15], which contains a generalization of Steinberg cross section theorem in this case.

To our aim, we exploit Sevostyanov's result together with the well-understood behaviour of spherical conjugacy classes with respect to the Bruhat decomposition. We replace the Slodowy slice by a suitable subset $\mathcal{S}_{w}$ of a Bruhat double coset $B w B$, depending on the stratum, such that its intersection with each given sheet in the stratum coincides (up to conjugation) with the intersection of the sheet with Sevostyanov's slice. Since for spherical conjugacy classes the intersection with this double coset is precisely the dense $B$-orbit, we show that the intersection of $\mathcal{S}_{w}$ with each conjugacy class is a single orbit for a finite 2-subgroup of a fixed maximal torus $T$.

Thanks to Sevostyanov's transversality result, a sheet $S$ of spherical classes is smooth if and only if $S \cap \mathcal{S}_{w}$ is also, and similarly for strata. This result is applied in Section 4 where we obtain the second main result of this paper: sheets containing a spherical class in simple groups are all smooth. As a consequence, we classify smooth strata of spherical classes in simple groups.

\section{Notation}

Unless otherwise stated, $G$ is a connected, reductive algebraic group over an algebraically closed field $k$ of good characteristic, i.e., not bad for any simple component of $[G, G]$.

Let $T$ be a fixed maximal torus of $G$ and let $\Phi$ be the associated root system. The root subgroup of $G$ associated with $\alpha \in \Phi$ will be denoted by $X_{\alpha}$ and $x_{\alpha}: k \rightarrow X_{\alpha}$ will indicate a parametrization of $X_{\alpha}$.

The Weyl group of $G$ will be denoted by $W$. The centralizer of an element $x \in G$ in a subgroup $H$ of $G$ will be denoted by $H^{x}$. The identity component of any subgroup $H$ of $G$ will be denoted by $H^{\circ}$.

Let $G$ act regularly on an irreducible variety $X$. For $n \geq 0$, we shall denote by $X_{(n)}$ the locally closed subset $X_{(n)}=\{x \in X \mid \operatorname{dim} G \cdot x=n\}$. For a subset $Y \subset X$, if $m$ is the maximum integer $n$ for which $Y \cap X_{(n)} \neq \emptyset$, the open subset $X_{(m)} \cap Y$ will be denoted by $Y^{r e g}$. A sheet for the action of $G$ on $X$ is an irreducible component of some $X_{(n)}$. We will investigate the case in which $X=G$ and the 
action is by conjugation. Sheets in this situation are related to Jordan classes (or decomposition classes) in $G$.

Recall that a Jordan class is an equivalence class for the following equivalence relation. Let $g=s u$ be the Jordan decomposition of $g \in G$. Then $g$ is equivalent to all elements $h \in G$ with Jordan decomposition $h=r v$ satisfying $G^{s \circ}=G^{r \circ}$, $r \in Z\left(G^{s \circ}\right)^{\circ} s$ and $v \in G^{s \circ} \cdot u$, and to any conjugate of any such $h$. In particular, Jordan classes consist of elements whose connected centralizers are all conjugate. As a set, the Jordan class $J(g)$ of $g$ is $J(g)=J(s u)=G \cdot\left(\left(Z\left(G^{s \circ}\right)^{\circ} s\right)^{r e g} u\right)$.

It has been shown in 8 that for any sheet $S$ there is a unique Jordan class $J=J(s u)$ such that $S=\bar{J}^{r e g}$. As a set,

$$
S=\bigcup_{z \in Z\left(G^{s \circ}\right)^{\circ}} G \cdot\left(z s \operatorname{Ind}_{G^{s \circ}}^{G^{z s \circ}} G^{s \circ} \cdot u\right)
$$

where $s$ and $u$ are as above and Ind denotes Lusztig-Spaltenstein induction [21]: for $L$ a Levi subgroup of a parabolic subgroup $P=L P^{u}$ of a reductive group $H$ and $L \cdot v$ a unipotent conjugacy class in $L, \operatorname{Ind}_{L}^{H}(L \cdot v)$ is the unique unipotent class of $H$ intersecting $v P^{u}$ in a dense set. We observe here that although $G^{s \circ}$ as in (2.1) might not be a Levi subgroup of a parabolic subgroup of $G$, it is always a Levi subgroup of a parabolic subgroup of $G^{z s \circ}$.

Sheets are related to the parts, called strata, of a partition defined in [20]. Strata are constructed as the non-empty fibers of a map $f$ from $G$ to the set of isomorphism classes of complex irreducible representations of $W$ which is given as follows. For $g=s u$, we have that $f(g u)=j_{W_{s}}^{W} \rho_{u}^{W_{s}}$, where $\rho_{u}^{W_{s}}$ is the irreducible Springer representation of the Weyl group $W_{s}$ of $G^{s \circ}$ associated with the unipotent class $G^{s \circ}$. $u$ and trivial local system and $j_{W_{s}}^{W}$ is Lusztig-Spaltenstein's truncated induction [21]. It was shown in [6] that, due to the compatibility of Lusztig-Spaltenstein truncated induction with induction of unipotent classes, $f$ is constant along sheets, strata are locally closed, and sheets of conjugacy classes are their irreducible components. For this reason we will work with sheets and strata simultaneously.

For a Borel subgroup $B \supset T$ and a conjugacy class $\mathcal{O}$ (a sheet $S$, respectively) in $G$, let $w_{\mathcal{O}}\left(w_{S}\right.$, respectively) be the unique element in $W$ such that $\mathcal{O} \cap B w_{\mathcal{O}} B$ is dense in $\mathcal{O}\left(S \cap B w_{S} B\right.$ is dense in $S$, respectively). If a sheet $S$ contains a spherical conjugacy class then $w_{S}=w_{\mathcal{O}}$ for every $\mathcal{O} \subset S$, [6, Proposition 5.3]. In addition, it follows from [6, Theorem 5.8] that $w_{S}$ is constant along strata containing spherical classes. The element $w_{\mathcal{O}}$ is always an involution and it is maximum in its conjugacy class with respect to the Bruhat ordering $([7,9])$.

The conjugacy classes of $w_{\mathcal{O}}$ and $w_{S}$ in $W$ are independent of the choice of a Borel subgroup containing $T$. Thus, the map $\mathcal{O} \mapsto w_{\mathcal{O}}$ determines a map $\varphi$ from the set of conjugacy classes in $G$ to the set of conjugacy classes of involutions in $W$.

For $w$ an involution in $W$, let $T^{w}:=\{t \in T \mid w(t)=t\}$, and $T_{w}:=\{t \in$ $\left.T \mid w(t)=t^{-1}\right\}$. Then $T=\left(T^{w}\right)^{\circ}\left(T_{w}\right)^{\circ}$ and $R_{w}:=T_{w} \cap T^{w}$ is an elementary abelian 2-group. For any choice of a Borel subgroup $B$ containing $T$ a longest element $w_{0} \in W$ is determined. Let $U$ be the unipotent radical of $B$. We set $U^{w}:=U \cap w^{-1} w_{0} U w_{0}^{-1} w$ and $U_{w}:=U \cap w^{-1} U w$. 


\section{Spherical Classes and Bruhat DECOMposition}

In this section we prove a Katsylo theorem for sheets containing spherical conjugacy classes. We will make use of the following general results.

Lemma 3.1 ([16, Lemma 2.13]). Let $A, B, C$ be varieties, let $\eta: A \rightarrow B$ be a smooth and surjective morphism and let $\theta: B \rightarrow C$ be a set-theoretic map such that $\theta \eta: A \rightarrow C$ is a morphism. Then $\theta$ is a morphism.

Lemma 3.2. Let $X$ be a $G$-variety and assume that there is an affine closed subset $\Sigma \subset X$ with an action of a finite group $\Gamma$ such that the following properties hold:

(1) for every $G$-orbit $\mathcal{O}$ of $X$ the set $\Sigma \cap \mathcal{O}$ is a $\Gamma$-orbit;

(2) the natural map $\mu: G \times \Sigma \rightarrow X$ is smooth and surjective.

Then, $X / G$ exists and it is isomorphic to $\Sigma / \Gamma$.

Proof. Define the map $\psi: X \rightarrow \Sigma / \Gamma$ set theoretically sending an element $x \in X$ to $G \cdot x \cap \Sigma$. We note that $\psi \mu: G \times \Sigma \rightarrow \Sigma / \Gamma$ is the composition of the natural projection on the second factor followed by the projection to the quotient. Therefore Lemma 3.1 applies with $A=G \times \Sigma, B=X, C=\Sigma / \Gamma \eta=\mu$ and $\theta=\psi$, so $\psi$ is a morphism. Since $\mu$ is surjective, $\Sigma$ meets all the $G$-orbits in $X$. The map $\psi$ is universally submersive because its restriction to $\Sigma$ is the canonical universal quotient map $\pi$ which is universally submersive [22, Theorem 1.1]. We now prove that for any open subset $V \subset \Sigma / \Gamma$ we have $\mathcal{O}_{\Sigma / \Gamma}(V) \simeq \mathcal{O}_{X}\left(\psi^{-1}(V)\right)^{G}$. It is enough to prove it for $V$ affine. For $f$ a morphism, let $f^{\#}$ be the map induced by $f$ on regular functions. Note that $\psi^{\#}$ embeds $k[V]$ into $k\left[\psi^{-1}(V)\right]^{G}$. Furthermore, if $\iota$ is the inclusion of $\Sigma$ in $X$, then $\iota^{\#}$ induces an embedding of $k\left[\psi^{-1}(V)\right]^{G}$ into $k\left[\pi^{-1}(V)\right]^{\Gamma}$. Since $\pi^{\#}=\iota^{\#} \circ \psi^{\#}$ induces an isomorphism $k[V] \simeq k\left[\pi^{-1}(V)\right]^{\Gamma}$, also $\iota^{\#}$ induces an isomorphism $k\left[\psi^{-1}(V)\right]^{G} \simeq k\left[\pi^{-1}(V)\right]^{\Gamma}$, hence $\psi^{\#}$ induces an isomorphism $k[V] \simeq k\left[\psi^{-1}(V)\right]^{G}$.

Let $\dot{w}$ be a representative of $w \in W$ in $N(T)$, let $B$ be a Borel subgroup containing $T$ and $U$ the unipotent radical of $B$. We define $\mathcal{S}_{w}:=\dot{w} T^{w} U^{w}$. Although $T^{w}$ and $U^{w}$ depend only on $w$ and not on its representative $\dot{w}$, the closed set $\mathcal{S}_{w}$ depends on the choice of the element $\dot{w}$ or, more precisely, on its coset $\dot{w} T^{w}$ in $T / T^{w}$. To keep notation simple, we omit reference to the specific representative in the symbol $\mathcal{S}_{w}$ as it will be clear from the context.

Lemma 3.3. Let $S$ be either a sheet or a stratum consisting of spherical conjugacy classes. Then $S \cap \mathcal{S}_{w_{S}}$ is a closed subset of $G$.

Proof. We show that $S \cap \mathcal{S}_{w_{S}}=\bar{S} \cap \mathcal{S}_{w_{S}}$. Let $\mathcal{O}$ be a conjugacy class in $S$. Then, $\operatorname{dim} \mathcal{O}=\ell\left(w_{\mathcal{O}}\right)+\operatorname{rk}\left(1-w_{\mathcal{O}}\right)$ by $\left[3,4,6,10,19\right.$. Since $w_{\mathcal{O}}=w_{S}$, we have $S \subset G_{(M)}$ where $M=\ell\left(w_{S}\right)+\operatorname{rk}\left(1-w_{S}\right)$ and $\bar{S} \backslash S \subset \bigcup_{m<M} G_{(m)}$; see [8, Proposition 5.1] and [6, Theorem 2.1]. If for some class $\mathcal{O}^{\prime}$ in $G$ and some $w \in W$ there holds $\mathcal{O}^{\prime} \cap B w B \neq \emptyset$, then $\operatorname{dim} \mathcal{O}^{\prime} \geq \ell(w)+\operatorname{rk}(1-w) ;[3$, Theorem 5]. Hence, if $\mathcal{O} \subset \bar{S} \backslash S$, then $\mathcal{O}^{\prime} \cap \mathcal{S}_{w_{S}} \subset \mathcal{O}^{\prime} \cap B w_{S} B=\emptyset$.

Let us briefly recall the construction of the closed subset $\Sigma_{w}$ of $G$ defined in [25, p. 1890], in the case in which $w \in W$ is an involution. Let $\mathfrak{h}_{\mathbb{R}}$ be the real span of the co-roots in the Cartan subalgebra $\mathfrak{h}$, let $\left\{v_{1}, \ldots, v_{r}\right\}$ be a basis of the $(-1)$ eigenspace of $w$ in $\mathfrak{h}_{\mathbb{R}}$, let $\mathfrak{h}_{\mathbb{R}}^{w}$ be the 1-eigenspace of $w$ in $\mathfrak{h}_{\mathbb{R}}$ and let $\Psi=\Phi \cap \mathfrak{h}_{\mathbb{R}}^{w}$. A set of positive roots $\Phi_{+}$can be defined according to the following rules: we choose a set of positive roots $\Psi_{+}$in the root system $\Psi$ and for $\beta \in \Psi$ we set $\beta \in \Phi_{+}$if 
and only if $\beta \in \Psi_{+}$. For $\beta \in \Phi \backslash \Psi$, as $w$ is an orthogonal involution, there will be a $j$ such that $\left(\beta, v_{j}\right) \neq 0$. Let us take $i$ maximal with this property. Then we have $\beta \in \Phi_{+}$if and only if $\beta\left(v_{i}\right)>0$.

Since $w\left(v_{i}\right)=-v_{i}$ for every $i$, there holds $\Phi_{+} \backslash \Psi=\left\{\alpha \in \Phi_{+} \mid w(\alpha) \in-\Phi_{+}\right\}$. In other words, with respect to the constructed choice of positive roots, $w$ has maximal possible length. In addition, $w\left(\Phi_{+} \backslash \Psi\right)=\left(-\Phi_{+}\right) \backslash \Psi$. Let U be the subgroup generated by the root subgroups corresponding to roots in $\Phi_{+}$and let $\mathrm{B}:=T \mathrm{U}$.

Let $\mathrm{w}$ be the unique representative of $w$ such that $\mathrm{w} x_{\alpha}(1) \mathrm{w}^{-1}=x_{w \alpha}(1)([12$, Theorem 5.4.2]), let $L$ be the Levi subgroup of a parabolic subgroup of $G$ containing $T$ and with root system $\Psi$, and let $P^{u}$ be the unipotent radical of the parabolic subgroup of $G$ containing all root subgroups associated with roots in $\left(-\Phi_{+}\right) \backslash \Psi$. Observe that $P^{u}$ is Sevostyanov's $N_{s}$ because $w$ is an involution and that ${ }_{\mathrm{w}} P^{u} \mathrm{w}^{-1}=$ $\mathrm{U}^{w}$. Sevostyanov's slice in this case is the closed subset

$$
\Sigma_{w}:=P^{u} L^{\mathrm{w}} \mathrm{w}=P^{u}{ }_{\mathrm{w}} L^{\mathrm{w}}=\mathrm{wU}^{w} L^{\mathrm{w}}=\mathrm{w}^{\mathrm{w}} \mathrm{U}^{w} .
$$

Lemma 3.4. Let $w \in W$ with $w^{2}=1$ and let $\dot{w}$ be a representative in $N(T)$. If $\mathcal{O}$ is a conjugacy class in $G$ such that $\mathcal{O} \cap B w B \neq \emptyset$, then $\mathcal{O} \cap \dot{w}\left(T^{w}\right)^{\circ} U \neq \emptyset$.

Proof. Clearly $\mathcal{O} \cap \dot{w} T U \neq \emptyset$. Let $x=\dot{w} t_{w} t^{w} u \in\left(T_{w}\right)^{\circ}\left(T^{w}\right)^{\circ} U$. Conjugation by $s \in\left(T_{w}\right)^{\circ}$ yields $\dot{w} s^{-2} t_{w} t^{w} u^{\prime} \in \mathcal{O}$. Since the square map on $\left(T_{w}\right)^{\circ}$ is onto, there exists $s \in\left(T_{w}\right)^{\circ}$ such that $s^{-2}=t_{w}$, whence the statement.

Lemma 3.5. Let $S$ be a stratum or a sheet containing a spherical conjugacy class $\mathcal{O}$. Let $w \in \varphi(\mathcal{O})$. For $\Phi_{+}, \mathrm{B}, \mathrm{U}$, w and $L$ as in the construction of Sevostyanov's slice and $\mathcal{S}_{w}=\mathrm{w} T^{w} \mathrm{U}^{w}, \Sigma_{w}={ }_{\mathrm{w}} L^{\mathrm{w}} \mathrm{U}^{w}$ we have

$$
\mathcal{O}^{\prime} \cap \mathcal{S}_{w}=\mathcal{O}^{\prime} \cap \Sigma_{w} \neq \emptyset
$$

for every class $\mathcal{O}^{\prime} \subset S$.

Proof. Let $\Delta_{+}$be the set of simple roots associated with $\Phi_{+}$and let $\Pi:=\Delta_{+} \cap \mathfrak{h}_{\mathbb{R}}^{w}$. With respect to the given choice of $\Phi_{+}$the element $w$ is of maximal length in $\varphi(\mathcal{O})$. Hence, it is equal to $w_{S}$ with respect to the choice of B. Therefore $\mathcal{O}^{\prime} \cap \mathrm{wB} \neq \emptyset$ for every $\mathcal{O}^{\prime} \subset S$ and, by Lemma 3.4. $\mathcal{O}^{\prime} \cap \mathrm{w} T^{w} \mathrm{U} \neq \emptyset$. By [4, we have $w=w_{0} w_{\Pi}$, for $w_{\Pi}$ the longest element in the parabolic subgroup $W_{\Pi}$ of $W$. Then, $L$ is the standard Levi subgroup of the standard parabolic subgroup $P_{\Pi}$ associated with $\Pi$, $\mathrm{U}_{w}=\mathrm{U}_{L}=\mathrm{U} \cap L$ and $\mathrm{U}^{w}=P_{\Pi}^{u}$.

We have $\mathcal{S}_{w}:=\mathrm{w}^{w} \mathrm{U}^{w} \subset \mathrm{w} L^{\mathrm{w}} \mathrm{U}^{w}=\Sigma_{w}$ so $S \cap \mathcal{S}_{w} \subset S \cap \Sigma_{w}$. Conversely, let $x=$ w $l u \in S \cap \mathrm{w} L^{\mathrm{w}} \mathrm{U}^{w}$. Then $l \in \mathrm{B} \sigma \mathrm{B}$ for some $\sigma \in W_{\Pi}$. Since $\ell\left(w_{0} w_{\Pi} \sigma\right)=\ell\left(w_{0} w_{\Pi}\right)+\ell(\sigma)$ for every $\sigma \in W_{\Pi}$, we have $x \in \mathrm{B} w \sigma \mathrm{B} \cap S$. Maximality of $w_{S}=w$ among all $\tau$ in $W$ such that $S \cap \mathrm{B} \tau \mathrm{B} \neq \emptyset$ forces $\sigma=1$. Hence, $l \in \mathrm{B} \cap L^{\mathrm{w}}=T^{w} \mathrm{U}_{w}$ and $S \cap \Sigma_{w} \subset \mathrm{w} T^{w} \mathrm{U}$. Let $\mathcal{O}^{\prime} \subset S$ and let $y=$ wtu $\in \mathrm{w} T \mathrm{U} \cap \mathcal{O}^{\prime}$. By [4, Lemmata 4.6, 4.7, 4.8] the only root subgroups occurring in the expression of $u$ are orthogonal to $\Pi$. Hence they lie in $\mathrm{U}^{w}$ and $\emptyset \neq \mathcal{O}^{\prime} \cap \mathrm{w} T^{w} \mathrm{U}=\mathcal{O}^{\prime} \cap \mathrm{w} T^{w} \mathrm{U}^{w}=\mathcal{O}^{\prime} \cap \Sigma_{w}=\mathcal{O}^{\prime} \cap \mathcal{S}_{w}$.

Remark 3.6. The results contained in 44 and needed in the proof of Lemma 3.3 refer to characteristic zero or odd and good. However, the proofs of Lemmata 4.6, 4.7, 4.8 and Theorems 2.7 and 4.4 therein are still valid for groups of type $A_{n}$ in characteristic 2 because also in this case spherical conjugacy classes meet only Bruhat cells corresponding to involutions in the Weyl group. This follows from [10, Theorem 3.4] and [11, Theorems 4.6, 4.7]. 
For an involution $w \in W$ let

$$
\Gamma_{w}:=\left\{t \in\left(T_{w}\right)^{\circ} \mid t^{2} \in T^{w}\right\}=\left\{t \in T_{w} \mid t^{2} \in R_{w}\right\} .
$$

Theorem 3.7. Let $S$ be a stratum or a sheet containing a spherical conjugacy class $\mathcal{O}$. Let $w \in \varphi(\mathcal{O})$. Then $S / G \simeq\left(S \cap \Sigma_{w}\right) / \Gamma_{w}$.

Proof. We apply Lemma 3.2 with $X=S, \Sigma=S \cap \Sigma_{w}$ and $\Gamma=\Gamma_{w}$.

By Lemmata 3.3 and 3.5 the set $\Sigma$ is affine and closed in $S$. The action of $\Gamma_{w}$ by conjugation preserves $\Sigma_{w}$, hence it preserves $\Sigma$.

Let $\mathcal{O}$ be a conjugacy class in $S$. We consider $\mathcal{O} \cap \Sigma=\mathcal{O} \cap \mathrm{w} T^{w} \mathrm{U} \subset \mathrm{B} w \mathrm{~B}$. Since $\mathcal{O}$ is spherical and $w=w_{S}=w_{\mathcal{O}}$, the set $\emptyset \neq \mathcal{O} \cap \mathrm{B} w \mathrm{~B}$ is the dense Borbit in $\mathcal{O}$ by [3, Theorem 5]. Therefore, for any $x=\mathrm{w} t_{x} u_{x}, y=\mathrm{w} t_{y} u_{y} \in \mathcal{O} \cap \Sigma$ there is $b=u v s_{0} s_{1} \in \mathrm{U}^{w} \mathrm{U}_{w}\left(T^{w}\right)^{\circ}\left(T_{w}\right)^{\circ}$ such that $u v s_{0} s_{1} \mathrm{w} t_{x} u_{x}=\mathrm{wt}_{y} u_{y} u v s_{0} s_{1}$. Since $v s_{0} s_{1} \mathrm{w} \in \mathrm{w} T \mathrm{U}$, uniqueness of the Bruhat decomposition $\mathrm{U}^{w} w T \mathrm{U}$ forces $u=1$, so $b=v s_{0} s_{1} \in \mathrm{U}_{w}\left(T^{w}\right)^{\circ}\left(T_{w}\right)^{\circ}$. In addition, $\left(T^{w}\right)^{\circ} \mathrm{U}_{w}$ centralizes all elements in $S \cap \mathrm{w} T^{w} \mathrm{U}^{w}$ ([4, Lemmata 4.6, 4.7, 4.9]), so $y=b x b^{-1}=s_{1} x s_{1}^{-1}$, that is, w $s_{1}^{-1} t_{x} u_{x}=$ ${ } t_{y} s_{1}\left(s_{1}^{-1} u_{y} s_{1}\right)$. This implies that $s_{1}^{2}=t_{x} t_{y}^{-1} \in T^{w}$ so $s_{1} \in \Gamma_{w}$.

The map $G \times \Sigma_{w} \rightarrow G$ is smooth by [25, Proposition 2.3]. The pull-back of this map along the inclusion $S \rightarrow G$ is the map $\mu$, and [14, Theorem III 10.1] applies.

Theorem 3.8. Let $S$ be a stratum or a sheet containing a spherical conjugacy class $\mathcal{O}$. Let $B=T U$ be a Borel subgroup of $G$, corresponding to a system of positive roots $\Phi^{+}$and a set of simple roots $\Delta$. Then, for any representative $\dot{w}_{S}$ of $w_{S}$ we have

$$
S / G \simeq\left(S \cap \mathcal{S}_{w_{S}}\right) / \Gamma_{w_{S}} .
$$

Proof. If $B=\mathrm{B}$, and $\dot{w}_{S}=\mathrm{w}$ as in the construction of Sevostyanov's slice, this is Theorem 3.7 in force of Lemma 3.5.

Let us assume that $\Delta_{+} \neq \Delta$ and let $\sigma \in W$ such that $\sigma \Delta=\Delta_{+}$. Then, $w_{S}^{\prime}:=\sigma w_{S} \sigma^{-1}$ is the maximum with respect to the Bruhat ordering determined by $\Delta_{+}$, i.e., $\mathrm{B} w_{S}^{\prime} \mathrm{B} \cap S$ is dense in $S$. Let $\dot{\sigma} \in N(T)$ be a representative of $\sigma$. Then $\dot{\sigma} T^{w_{S}} \dot{\sigma}^{-1}=T^{w_{S}^{\prime}}$ and $\dot{\sigma} U^{w_{S}} \dot{\sigma}^{-1}=\mathrm{U}^{w_{S}^{\prime}}$. In addition $\dot{\sigma} \dot{w}_{S} \dot{\sigma}^{-1} \in \mathrm{w}_{S}^{\prime}\left(T^{w_{S}}\right)^{\circ}\left(T_{w_{S}}\right)^{\circ}$, where $\mathrm{w}_{S}^{\prime}$ is the representative needed for Sevostyanov's construction. Up to multiplying $\dot{\sigma}$ by a suitable element in $\left(T_{w_{S}}\right)^{\circ}$, we can make sure that $\dot{\sigma} \dot{w}_{S} \dot{\sigma}^{-1} \in$ $\mathrm{w}_{S}^{\prime}\left(T^{w_{S}}\right)^{\circ}$ so $\dot{\sigma} \dot{w}_{S} T^{w_{S}} U^{w_{S}} \dot{\sigma}^{-1}=\mathrm{w}_{S}^{\prime}\left(T^{w_{S}}\right)^{\circ} \mathrm{U}^{w_{S}^{\prime}}$. So, conjugation by $\dot{\sigma}$ maps $S \cap \mathcal{S}_{w_{S}}$

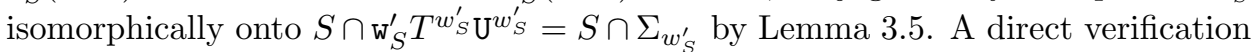
shows that $\dot{\sigma} \Gamma_{w_{S}} \dot{\sigma}^{-1}=\Gamma_{w_{S}^{\prime}}$, whence the statement follows from Theorem 3.7 .

\section{Smoothness of SheEts of SPHerical Classes}

In this section we detect when $S$ is smooth, for $S$ a sheet or a stratum of spherical classes in a simple group $G$. By [8, Remark 3.4, Proposition 5.1] it is enough to consider a representative for each isogeny class of $G$. For $G$ of classical type we will consider matrix groups, whereas for $G$ of exceptional type we shall consider the simply-connected group. We will use the classification of spherical conjugacy classes in [5].

Arguing as in [16] (see also [1, Proposition 3.9 (iii)]), we conclude that $S$ is smooth if and only if $S \cap \mathcal{S}_{w}$ is also. We analyze smoothness of the latter. In order to do so, we recall some information contained in [4, Lemmata 4.6, 4.7, 4.8] and Remark 3.6 about this intersection. 
Let $w_{S}$ be the Weyl group element associated with $S$. We recall that it always has the form $w_{S}=w_{0} w_{\Pi}$ for some $\Pi \subset \Delta$ and that $w_{S}(\alpha)=\alpha$ for every $\alpha \in \Pi$; see Lemma 3.5. Let $\dot{w}_{S}$ be a representative of $w_{S}$ such that $\dot{w}_{S} x_{\alpha}(\xi) \dot{w}_{S}^{-1}=x_{\alpha}(\xi)$ for every $\xi \in k$ and every $\alpha \in \Phi \cap \mathbb{Z} \Pi$. Let $\mathcal{O}$ be a class in $S$. If $\dot{w}_{S} t \mathrm{v} \in \mathcal{O} \cap \dot{w}_{S} T U$, then $v$ lies in $V_{S}:=\prod_{\beta \in \Phi^{+}, w_{S} \beta=-\beta} X_{\beta}$, where $X_{\beta}$ is the root subgroup associated with $\beta$. We observe that, due to the Chevalley commutator formula the product of subgroups can be taken in arbitrary order and that $V_{S}$ is a subgroup of $U$. In addition, $\dot{w}_{S} t$ commutes with $X_{\alpha}$ for every $\alpha \in \mathbb{Z} \Pi \cap \Phi$. Therefore,

$$
S \cap \mathcal{S}_{w_{S}} \subset \dot{w}_{S}\left(Z\left(L_{\Pi}\right) \cap T^{w_{S}}\right) V_{S} .
$$

We will make use of the following observation.

Proposition 4.1. Let $G$ be a simple algebraic group and let $S=\overline{J(s u)}^{\text {reg }}$ for some $s, u \in G$ be a sheet of spherical conjugacy classes in $G$. Then either $u=1$ or $S=G \cdot$ su and, if $S \neq G \cdot$ su, then $S$ contains a semisimple and a unipotent element.

Proof. By the classification of spherical conjugacy classes in [3,5], if $G \cdot r v$ is spherical and $v \neq 1$, then $G^{r \circ}$ is semisimple. Therefore, either $G \cdot r v$ is a single sheet, or it lies in $S=\overline{J(s)}^{r e g}$, for some semisimple element $s$. In addition, if $S=\overline{J(s)}^{\text {reg }}$ is nontrivial, then $G^{s \circ}$ is a Levi subgroup, so $S$ contains a unipotent class by [8, Theorem $5.6(\mathrm{~b})]$.

We can state the main result of this section.

Theorem 4.2. Let $G$ be a simple algebraic group over $k$.

(1) All sheets of spherical conjugacy classes are smooth.

(2) Let $S$ be a stratum of spherical conjugacy classes. Then $S$ is smooth with the following exceptions:

- $G$ is of type $B_{2}$ and $S$ is the stratum containing the unipotent class with partition $\left(3,1^{2}\right)$ or, equivalently, $G$ is of type $C_{2}$ and $S$ is the stratum containing the unipotent class with partition $\left(2^{2}\right)$;

- $G$ is of type $D_{2 h+1}$ and $S$ is the stratum containing the unipotent class with partition $\left(2^{2 h}, 1^{2}\right)$.

Proof. By Proposition 4.1 it is enough to look at sheets containing a semisimple element whose connected centralizer is not semisimple. Their description follows from (2.1) and the classification in [3,5]. For each simple group we will compute the set theoretical intersection of every non-trivial spherical sheet $S$ with the corresponding $\mathcal{S}_{w_{S}}$ and we will use the following remark.

Remark 4.3. The intersection of $S \cap \mathcal{S}_{w_{S}}$ is reduced. This is proved through the following steps:

(1) $S$ is reduced.

(2) The map $G \times\left(\mathcal{S}_{w_{S}} \cap S\right) \rightarrow S$ is smooth and surjective, which follows from [25. Proposition 2.3] and [14, III, Theorem 10.1].

(3) $G \times\left(\mathcal{S}_{w_{S}} \cap S\right)$ is reduced, which follows from the previous facts using [13, éxp. II, prop.3.1].

(4) $G \times\left(\mathcal{S}_{w_{S}} \cap S\right)$ is reduced implies that $\left(\mathcal{S}_{w_{S}} \cap S\right)$ is reduced. 
4.1. Type $A_{n}$. Let us first consider $H=G L_{n+1}(k)$. We choose $B$ to be the subgroup of upper triangular matrices, and $T$ to be the subgroup of diagonal matrices. In this case sheets and strata coincide, spherical sheets are parametrized by $m=0, \ldots,\left[\frac{n+1}{2}\right]$ and they are as follows: $S_{m}=Z(H) \mathcal{O}_{m} \cup \bigcup_{\lambda, \mu \in k^{*} ; \lambda \neq \mu} \mathcal{O}_{m}(\lambda, \mu)$, where $\mathcal{O}_{m}$ is the unipotent class corresponding to the partition $\left(2^{m}, 1^{n+1-2 m}\right)$ and $\mathcal{O}_{m}(\lambda, \mu)$ is the semisimple class with eigenvalues $\lambda$ with multiplicity $n+1-2 m$ and $\mu$ with multiplicity $2 m$, the case $m=0$ being trivial. The Weyl group element associated to $S_{m}$ is $w_{0} w_{\Pi}$ where $\Pi=\left\{\alpha_{m+1}, \alpha_{m+2}, \ldots, \alpha_{n+1-m}\right\}$. We choose $\dot{w}_{S_{m}}=\left(\begin{array}{ccc}0 & 0 & J_{m} \\ 0 & I_{n+1-2 m} & 0 \\ -J_{m} & 0 & 0\end{array}\right)$ where $J_{m}$ is the $m \times m$ matrix with 1 on the antidiagonal and 0 elsewhere. Then

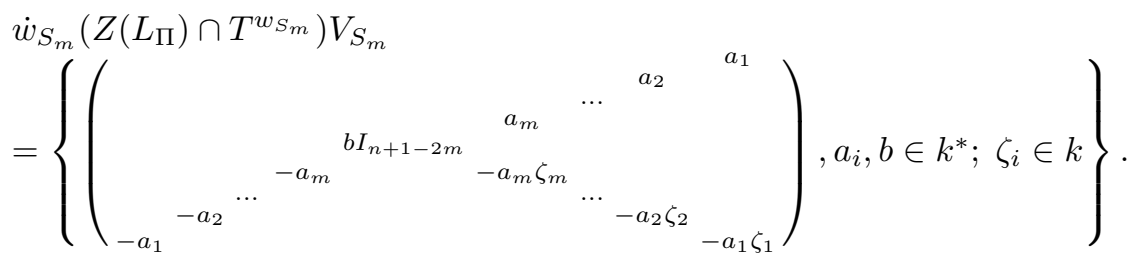

A matrix in $\dot{w}_{S_{m}}\left(Z\left(L_{\Pi}\right) \cap T^{w_{S_{m}}}\right) V_{S_{m}}$ lies in $S_{m}$ if either all its eigenvalues are equal or else it has two eigenvalues and it is semisimple. This happens if and only if

$$
\begin{aligned}
& \operatorname{Tr}\left(\begin{array}{cc}
0 & a_{i} \\
-a_{i} & -a_{i} \zeta_{i}
\end{array}\right)=\operatorname{Tr}\left(\begin{array}{cc}
0 & a_{j} \\
-a_{j} & -a_{j} \zeta_{j}
\end{array}\right) \quad \forall i, j, \\
& \operatorname{det}\left(\begin{array}{cc}
0 & a_{i} \\
-a_{i} & -a_{i} \zeta_{i}
\end{array}\right)=\operatorname{det}\left(\begin{array}{cc}
0 & a_{j} \\
-a_{j} & -a_{j} \zeta_{j}
\end{array}\right) \quad \forall i, j, \\
& \operatorname{det}\left(\begin{array}{cc}
-b & a_{1} \\
-a_{1} & -a_{1} \zeta_{1}-b
\end{array}\right)=0,
\end{aligned}
$$

that is, if and only if there exist $\epsilon_{2}, \ldots, \epsilon_{m} \in\{0,1\}$ such that $a_{i}=\epsilon_{i} a_{1}, \zeta_{i}=\epsilon_{i} \zeta_{1}$ and $\zeta_{i}=-a_{1}^{2} b^{-1}-b^{2} a_{1}^{-1}$. The set theoretic intersection $S_{m} \cap \mathcal{S}_{\dot{w}_{S_{m}}}$ is then a union of $(m-1)$ disjoint irreducible components each isomorphic to the image of the morphism

$$
\begin{aligned}
f: k^{*} \times k^{*} & \rightarrow k^{*} \times k^{*} \times k, \\
(a, b) & \mapsto\left(a, b, a^{2} b^{-1}+b\right) .
\end{aligned}
$$

Being a graph, this intersection is smooth for every field $k$ and every $m$. By Remark 4.3. this intersection coincides with the scheme theoretic one.

Let us now consider $G=S L_{n}(k)$. Set theoretically, every sheet of spherical classes $S$ is contained in the intersection of some $S_{m}$ with $G$. If $\operatorname{char}(k)=p$ does not divide $n+1$, then $S \cap \mathcal{S}_{w_{S_{m}}}$ is contained in the image through $f$ of the disjoint smooth curves $\mathcal{C}_{ \pm 1}$ of equation $a^{2 m} b^{n+1-2 m}- \pm 1=0$. By Remark 4.3, this set theoretic inclusion is scheme theoretic hence $S \cap \mathcal{S}_{w_{S_{m}}}$ is smooth.

Let us now assume that $p \mid n+1$. Then, for every $m$ coprime with $p$, the argument above applies. If, instead, $p \mid m$ then we still have the set-theoretical inclusion $S \cap$ $\mathcal{S}_{w_{S}} \subset f\left(\mathcal{C}_{1} \cup \mathcal{C}_{-1}\right)$ but the curves $\mathcal{C}_{ \pm 1}$ are not reduced. The reduced scheme of $f\left(\mathcal{C}_{1} \cup \mathcal{C}_{-1}\right)$ is smooth and the above argument applies.

4.2. Type $B_{n}$. Let $G=S O_{2 n+1}(k)$ with $n \geq 2$. We realize it as the subgroup of matrices in $S L_{2 n+1}(k)$ leaving invariant the symmetric bilinear form whose associated matrix with respect to the canonical basis of $k^{n}$ is $\left(\begin{array}{ccc}1 & 0 & 0 \\ 0 & 0 & I_{n} \\ 0 & I_{n} & 0\end{array}\right)$. We choose $T$ to be its subgroup of diagonal matrices and $B$ to be the subgroup of matrices of 
the form $\left(\begin{array}{ccc}1 & 0 & t_{v} \\ -A v & A & A M \\ 0 & 0 & { }^{t} A^{-1}\end{array}\right)$ where $A$ is an upper-triangular matrix in $G L_{n}(k), v$ is a column vector in $k^{n}, M=(-1 / 2) v^{t} v+\Sigma, \Sigma$ is a skew-symmetric matrix of size $n$ and ${ }^{t} X$ denotes the transpose of $X$, for any matrix $X$.

The non-trivial sheets of spherical conjugacy classes are given by $S$ and $S^{\prime}$, with

$$
S=\left(\bigcup_{\lambda \neq 0, \pm 1} \mathcal{O}_{\lambda}\right) \cup \mathcal{O}_{(3,2)} \cup G \cdot \rho_{n} u
$$

where $\mathcal{O}_{\lambda}$ is the semisimple class with eigenvalues $1, \lambda, \lambda^{-1}$ with multiplicity $1, n, n$ respectively, $\mathcal{O}_{(3,2)}$ is the unipotent conjugacy class corresponding to the partition $\left(3,2^{n-1}\right)$, for $n$ odd and $\left(3,2^{n-2}, 1^{2}\right)$ for $n$ even, the element $\rho_{n}$ is the diagonal matrix $\operatorname{diag}\left(1,-I_{2 n}\right)$ and $u$ is a representative of any unipotent conjugacy class in $G^{\rho_{n} \circ} \cong S O_{2 n}(k)$ associated with the partition $\left(2^{n}\right)$ when $n$ is even, and $\left(2^{n-1}, 1^{2}\right)$ when $n$ is odd, and

$$
S^{\prime}=\left(\bigcup_{\lambda \neq 0,1} \mathcal{O}_{\lambda, 1}\right) \cup \mathcal{O}_{\left(3,1^{2 n-2}\right)},
$$

where $\mathcal{O}_{\lambda, 1}$ is the class of a semisimple matrix with eigenvalues $1, \lambda, \lambda^{-1}$ with multiplicity $2 n-1,1,1$, respectively and $\mathcal{O}_{\left(3,1^{2 n-2}\right)}$ is the unipotent conjugacy class with associated partition $\left(3,1^{2 n-2}\right)$.

We have $S \cap S^{\prime}=\emptyset$ unless $n=2$, so the stratum containing $S$ is not smooth for $n=2$ whereas for $n \geq 3$ the strata containing $S$ and $S^{\prime}$ are smooth if and only if $S$ and $S^{\prime}$ are so.

Let us analyze $S$. Here, $w_{S}=w_{0}$. If we choose $\dot{w}_{S}=\left(\begin{array}{ccc}(-1)^{n} & 0 & 0 \\ 0 & 0 & I_{n} \\ 0 & I_{n} & 0\end{array}\right)$, then $\mathcal{S}_{w_{S}}=\dot{w}_{S} T^{w_{S}} U$ consists of matrices of the form

$$
X=X(E, M, Q, v)=\left(\begin{array}{ccc}
(-1)^{n} & 0 & (-1)^{n}{ }^{t} v \\
0 & 0 & E^{t} Q^{-1} \\
-E Q v & E Q & E Q M
\end{array}\right)
$$

where $E \in\{ \pm 1\}^{n}, v={ }^{t}\left(v_{1}, \ldots, v_{n}\right) \in k^{n}, Q$ is a unipotent upper triangular matrix in $G L_{n}(k)$, and $M=(-1 / 2) v^{t} v+A$, where $A$ is skew-symmetric.

Now, if $X$ lies in $S$ then there exists $\lambda \in k^{*}$ such that $\operatorname{rk}(X-\lambda I) \leq n+1$.

Assume first that $X=X(E, M, Q, v)$ satisfies $\operatorname{rk}(X-\lambda I) \leq n+1$ for some $\lambda \neq(-1)^{n}$. Then we have

$$
M-\lambda Q^{-1} E+\lambda^{-1} E^{t} Q^{-1}+\frac{(-1)^{n}}{(-1)^{n}-\lambda} v^{t} v=0 .
$$

Let $\varphi_{\lambda, n}:=\frac{(-1)^{n}+\lambda}{2\left((-1)^{n}-\lambda\right)}$. Taking symmetric and skew-symmetric parts in (4.1) we obtain the following equations:

$$
\varphi_{\lambda, n} v^{t} v=(1 / 2)\left(\lambda-\lambda^{-1}\right)\left(Q^{-1} E+E^{t} Q^{-1}\right)
$$

and

$$
A=(1 / 2)\left(\lambda+\lambda^{-1}\right)\left(Q^{-1} E-E^{t} Q^{-1}\right) .
$$

The diagonal terms in (4.1) give

$$
\varphi_{\lambda, n} e_{i} v_{i}^{2}=\left(\lambda-\lambda^{-1}\right) .
$$

Hence, if in addition $\lambda \neq(-1)^{n+1}$, then $e_{i} v_{i}^{2}=e_{1} v_{1}^{2}$ for every $i$. We fix, for $i=1, \ldots, n$, elements $\zeta_{i} \in k$ such that $\zeta_{i}^{2}=e_{i}$ and we set $a_{\lambda}:=\zeta_{1} v_{1}$, so $\varphi_{\lambda, n} a_{\lambda}^{2}=$ 
$\left(\lambda-\lambda^{-1}\right)$. Therefore, for every $j \geq 1$ there is $\eta_{j}= \pm 1$, with $\eta_{1}=1$, such that $\zeta_{j} v_{j}=\eta_{j} a_{\lambda}$. Thus, we have

$$
\lambda^{2}-\left(2(-1)^{n}-a_{\lambda}^{2} / 2\right) \lambda+1=0
$$

which gives

$$
\left(\lambda+\lambda^{-1}\right)=\left(2(-1)^{n}-a_{\lambda}^{2} / 2\right) .
$$

Making use of (4.4) and (4.6), for $2 \leq i<j \leq n$, the $(i, j)$ entries of (4.2) and (4.3) give

$$
\left(Q^{-1}\right)_{i j}=2 \zeta_{i}^{-1} \zeta_{j} \eta_{i} \eta_{j}, \quad a_{i j}=\left(2(-1)^{n}-a_{\lambda}^{2} / 2\right) \zeta_{i}^{-1} \zeta_{j}^{-1} \eta_{i} \eta_{j}
$$

So, for $\lambda \neq \pm 1$ and for every choice of $\eta_{i}, \zeta_{i}$, the matrix $Q$ is completely determined, the vector $v$ depends linearly on $a_{\lambda}$ and $M$ depends on $a_{\lambda}^{2}$, giving a dense subset of a line. Conversely, if $\lambda \neq \pm 1$, the condition $\operatorname{rk}(X-\lambda I) \leq n+1$ also implies that $X$ is semisimple, and it ensures $X \in S$.

Let us now assume $\lambda=(-1)^{n+1}$. Then, (4.3) gives $a_{i j}=(-1)^{n+1}\left(Q^{-1}\right)_{i j} e_{j}$ for every $i<j . X$ lies in $S$ only if $\operatorname{rk}\left(X-(-1)^{n+1}\right)^{2}=1$. Looking at the $(2,2)$-block in this matrix we get $\operatorname{rk}\left({ }^{t} Q^{-1} E+E Q^{-1}\right) \leq 1$, which yields

$$
\left(Q^{-1}\right)_{i j}=2 \zeta_{i}^{-1} \zeta_{j} \eta_{i} \eta_{j} \text { and } a_{i j}=2(-1)^{n+1} \zeta_{i}^{-1} \zeta_{j}^{-1} \eta_{i} \eta_{j}
$$

Let $N=\left(X-(-1)^{n+1}\right)^{2} \operatorname{diag}\left(1, Q^{-1} E, I_{n}\right)$. Every row in $N$ must be a multiple of the first one, which is non-zero. Thus, every row in the block $(2,2)$ must be a multiple of the $(1,2)$-block. This gives $e_{i} v_{i}^{2}=e_{1} v_{1}^{2} \neq 0$ for every $i \geq 0$. We set $a=\zeta_{1} v_{1}$, so for every $i \geq 2$ we have $v_{i}=a \zeta_{i}^{-1} \eta_{i}$. A direct computation shows that ${ }^{t} v E Q v=0$ and $-E^{t} Q^{-1} E Q v=v$. The condition that the principal minor of size 2 must be 0 gives $a^{2}=8(-1)^{n}$, i.e., $a$ satisfies condition (4.5) so (4.7) is verified also in this case. Thus, for the Jordan class $J$ of $\operatorname{diag}\left(1, \lambda I_{n}, \lambda^{-1} I_{n}\right)$, the set-theoretical intersection $J \cap \mathcal{S}_{w_{0}}$ is a disjoint union of $2^{2 n-1}$ copies of $k^{*}$, given by the values of $a_{\lambda}$, one for each choice of each $e_{j}$ 's and of the $\eta_{j}$ 's.

Let us now consider $\lambda=(-1)^{n}$. Then $X$ must satisfy the condition $\operatorname{rk}(X-$ $\left.(-1)^{n}\right)=n$, which forces $a_{\lambda}=0$. Moreover, $X$ lies in $S$ only if $\operatorname{rk}\left(X-(-1)^{n}\right)=1$, which, combined with (4.3) gives the condition $\operatorname{rk}\left({ }^{t} Q^{-1} E+E Q^{-1}\right) \leq 1$, which yields (4.7) with $a_{\lambda}=0$. The dimensional argument used in the proof of Lemma 3.3 for the $G$-conjugacy class of $X$ shows that $X \in S$. Hence the set theoretical intersection $S \cap \mathcal{S}_{w_{S}}$ is a disjoint union of $2^{2 n-1}$ copies of $k$. By Remark 4.3, this is also the scheme theoretic intersection so $S$ is smooth.

Let us now consider $S^{\prime}$. In this case $w_{S^{\prime}}=s_{\beta} s_{1}=w_{0} w_{\Pi}$ for $\Pi=\left\{\alpha_{3}, \ldots, \alpha_{n}\right\}$ and $\beta=\varepsilon_{1}+\varepsilon_{2}$ the highest long root. We choose $\dot{w}_{S^{\prime}}=\left(\begin{array}{ccccc}1 & 0 & 0 & 0 & 0 \\ 0 & 0 & 0 & I_{2} & 0 \\ 0 & 0 & I_{n-2} & 0 & 0 \\ 0 & I_{2} & 0 & 0 & 0 \\ 0 & 0 & 0 & 0 & I_{n-2}\end{array}\right)$ so

$$
\begin{aligned}
& \dot{w}_{S^{\prime}}\left(Z\left(L_{\Pi}\right) \cap T^{w_{S^{\prime}}}\right) V_{S^{\prime}}
\end{aligned}
$$

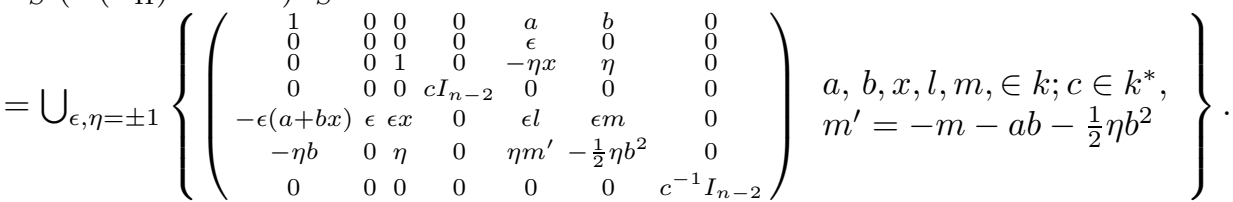

Then an element $X \in \dot{w}_{S^{\prime}}\left(Z\left(L_{\Pi}\right) \cap T^{w_{S^{\prime}}}\right) V_{S^{\prime}}$ lies in $S^{\prime}$ if and only if $r k(X-I)=2$ : this is clear if the eigenvalues different from 1 are distinct. If the eigenvalues different from 1 are equal to -1 , then this follows from the fact that the unipotent 
part must lie in the connected centralizer of the semisimple part. If the eigenvalues are all equal to 1 , then there are only two unipotent classes for which $\operatorname{rk}(X-I)=2$, namely the one associated with $\left(2^{2}, 1^{2 n-3}\right)$ and $\mathcal{O}_{\left(3,1^{2 n-3}\right)}$. For dimensional reasons, the former does not intersect $\mathcal{S}_{w_{s^{\prime}}}$.

Assume $r k(X-I)=2$. For such an $X$ we have

$$
c=1, \quad a=b=0, \quad l=\eta x^{2}, \quad m=-\eta x .
$$

By Remark 4.3 the variety $S^{\prime} \cap \mathcal{S}_{w_{S^{\prime}}}$ is isomorphic to a disjoint union of 4 affine lines, one for each value of $\eta$ and $\epsilon$.

4.3. Type $C_{n}$. Let us consider $G=S p_{2 n}(k)$ with $n \geq 3$. We realize it as the subgroup of matrices in $S L_{2 n}(k)$ leaving invariant the skew-symmetric bilinear form whose associated matrix with respect to the canonical basis of $k^{n}$ is $\left(\begin{array}{cc}0 & I_{n} \\ -I_{n} & 0\end{array}\right)$. We choose $T$ to be its subgroup of diagonal matrices and $B$ to be the subgroup of matrices of the form $\left(\begin{array}{cc}A & A \Sigma \\ 0 & t^{t} A^{-1}\end{array}\right)$ where $A$ is an upper-triangular matrix in $G L_{n}(k)$ and $\Sigma$ is a symmetric matrix of size $n$.

There are, up to a central element, two non-trivial sheets of spherical classes, $\pm S_{1}$ and $S_{2}$ where

$$
S_{1}=\left(\bigcup_{\lambda \neq 0, \pm 1} \mathcal{O}_{(\lambda, 1)}\right) \cup \mathcal{O}_{\left(2^{2}, 1^{2 n-4}\right)} \cup G \cdot \sigma_{1} x_{\beta}(1)
$$

where $\mathcal{O}_{(\lambda, 1)}$ is the semisimple class with eigenvalues $\lambda, \lambda^{-1}$ and 1 , with multiplicity $1,1,2 n-2$ respectively, $\mathcal{O}_{\left(2^{2}, 1^{2 n-4}\right)}$ is the unipotent conjugacy class corresponding to the partition $\left(2^{2}, 1^{2 n-4}\right)$, the element $\sigma_{1}$ is the diagonal matrix $\operatorname{diag}\left(-1, I_{n-1},-1, I_{n-1}\right)$ and $\beta=\varepsilon_{1}+\varepsilon_{2}$ is the highest root, and

$$
S_{2}=\left(\bigcup_{\lambda \neq 0, \pm 1} \mathcal{O}_{\lambda}\right) \cup \pm \mathcal{O}_{\left(2^{n}\right)}
$$

where $\mathcal{O}_{\lambda}$ is the semisimple class with eigenvalues $\lambda^{ \pm 1}$ and $\mathcal{O}_{\left(2^{n}\right)}$ is the unipotent conjugacy class corresponding to the partition $\left(2^{n}\right)$.

Since $n>2$, we always have $S_{1} \cap S_{2}=S_{2} \cap\left(-S_{1}\right)=\emptyset$, hence the strata containing these sheets are smooth if and only if the sheets are also.

The Weyl group element corresponding to $S_{1}$ is $w_{S_{1}}=w_{0} w_{\Pi_{1}}$, for $\Pi_{1}=\left\{\alpha_{3}, \ldots\right.$, $\left.\alpha_{n}\right\}$, so $w_{S_{1}}=s_{\alpha_{1}} s_{\beta}$. We choose $\dot{w}_{S_{1}}=\left(\begin{array}{cc}I_{2} I_{n-2} & \\ -I_{2} & \end{array}\right)$ so

$$
\begin{aligned}
& \dot{w}_{S_{1}}\left(Z\left(L_{\Pi_{1}}\right) \cap T^{w_{S_{1}}}\right) V_{S_{1}}
\end{aligned}
$$

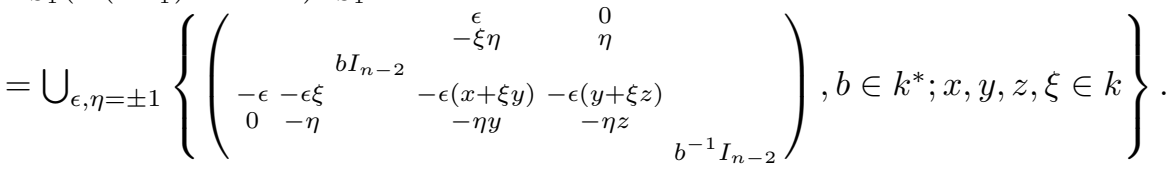

Then $X \in \dot{w}_{S_{1}}\left(Z\left(L_{\Pi_{1}}\right) \cap T^{w_{S_{1}}}\right) V_{S_{1}}$ lies in $S_{1}$ if and only if $r k(X-I)=2$, which holds if and only if

$$
b=1, \quad x=-2 \epsilon, \quad y=\eta \xi, \quad z=-2 \eta .
$$

By Remark 4.3 the variety $S_{1} \cap \mathcal{S}_{w_{S_{1}}}$ is isomorphic to a disjoint union of affine lines.

The Weyl group element corresponding to $S_{2}$ is $w_{0}$ and we choose the representative $\dot{w}_{0}=\left(\begin{array}{cc}0 & I_{n} \\ -I_{n} & 0\end{array}\right)$. Then the matrices in $\dot{w}_{0} T^{w_{0}} V_{S_{2}}$ are all matrices of the form 
$x(E, V, X)=\left(\begin{array}{cc}0 & E^{t} V^{-1} \\ -E V & -E V X\end{array}\right)$, where $E=\operatorname{diag}\left(\epsilon_{1}, \ldots, \epsilon_{n}\right), \epsilon_{i}= \pm 1$ for every $i, V$ is an upper triangular unipotent matrix, and $X$ is a symmetric matrix.

If $x(E, V, X)$ lies in $S_{2}$, then there is a $\lambda \in k^{*}$ for which $\operatorname{rk}(x(E, V, X)-\lambda I)=n$. This forces $\lambda X+\lambda^{2}\left(V^{-1} E\right)+{ }^{t}\left(V^{-1} E\right)=0$. If $\lambda^{2} \neq 1$ this can happen only if $V=I$ and $X=-\left(\lambda+\lambda^{-1}\right) E$ and $x\left(E, V,-\left(\lambda+\lambda^{-1}\right) E\right) \in \mathcal{O}_{\lambda}$. If instead $\lambda^{2}=1$, then $x(E, V, X)$ lies in $S_{2}$ only if $(x(E, V, X)-\lambda I)^{2}=0$. A direct computation shows that this is possible only if $V=I$, so $X=-2 \lambda^{-1} E=-\left(\lambda+\lambda^{-1}\right) E$. If this is the case, then $x\left(E, I,-2 \lambda^{-1} E\right) \in \lambda \mathcal{O}_{\left(2^{n}\right)}$. Therefore, set theoretically, $S_{2} \cap \mathcal{S}_{w_{0}}$ is a disjoint union of affine lines with coordinate $\lambda+\lambda^{-1}$. We apply Remark 4.3 .

4.4. Type $D_{n}$. Let $G=S O_{2 n}(k)$, for $n \geq 4$. We realize it as the subgroup of matrices in $S L_{2 n}(k)$ leaving invariant the symmetric bilinear form whose associated matrix with respect to the canonical basis of $k^{n}$ is $\left(\begin{array}{cc}0 & I_{n} \\ I_{n} & 0\end{array}\right)$. We choose $T$ to be its subgroup of diagonal matrices and $B$ to be the subgroup of matrices of the form $\left(\begin{array}{cc}A & A \Sigma \\ 0 & t^{t} A^{-1}\end{array}\right)$ where $A$ is an upper-triangular matrix in $G L_{n}(k)$ and $\Sigma$ is a skewsymmetric matrix of size $n$.

It is convenient to separate the cases of $n$ even and odd.

4.4.1. $D_{n}$ for $n$ even. Let $n=2 h$. Let $\theta$ be an automorphism of $G$ induced by the non-trivial automorphism of its Dynkin diagram interchanging the last two simple roots. The only non-trivial sheets of spherical conjugacy classes are given by $S$, $\theta(S)$ and $S^{\prime}$ as follows:

$$
S=\bigcup_{\lambda \neq 0, \pm 1}\left(G \cdot\left(\begin{array}{ll}
\lambda I_{n} & \\
& \lambda^{-1} I_{n}
\end{array}\right)\right) \cup \pm \mathcal{O}_{\left(2^{n}\right)}
$$

and of type

$$
\theta(S)=\bigcup_{\lambda \neq 0, \pm 1}\left(G \cdot\left(\begin{array}{lll}
\lambda I_{n-1} & & \\
& \lambda^{-1} I_{n} & \\
& & \lambda
\end{array}\right)\right) \cup \pm \mathcal{O}_{\left(2^{n}\right)}^{\prime}
$$

where $\mathcal{O}_{\left(2^{n}\right)}$ and $\mathcal{O}_{\left(2^{n}\right)}^{\prime}$ are the two distinct unipotent conjugacy classes corresponding to the partition $\left(2^{n}\right)$ and

$$
S^{\prime}=\left(\bigcup_{\lambda \neq 0,1} \mathcal{O}_{\lambda, 1}\right) \cup \mathcal{O}_{\left(3,1^{2 n-3}\right)}
$$

where $\mathcal{O}_{\lambda, 1}$ is the class of a semisimple matrix with eigenvalues $1, \lambda, \lambda^{-1}$ with multiplicity $2 n-2,1,1$, respectively, and $\mathcal{O}_{\left(3,1^{2 n-3}\right)}$ is the unipotent conjugacy class with associated partition $\left(3,1^{2 n-3}\right)$.

If $n=4$, then for any automorphism $\vartheta$ of $G$ induced by a Dynkin diagram automorphism corresponding to the cyclic permutation of the simple roots $\alpha_{1} \mapsto$ $\alpha_{3} \mapsto \alpha_{4} \mapsto \alpha_{1}$, we have $\vartheta(S)=S^{\prime}$. This can be seen by comparing the Jordan normal forms of $x_{\alpha_{1}}(\xi) x_{\alpha_{3}}(\eta) \in \mathcal{O}_{\left(2^{4}\right)}$ and $\vartheta\left(x_{\alpha_{1}}(\xi) x_{\alpha_{3}}(\eta)\right)=x_{\alpha_{3}}\left(\xi^{\prime}\right) x_{\alpha_{4}}\left(\eta^{\prime}\right)$, for $\xi, \xi^{\prime}, \eta, \eta^{\prime} \in k^{*}$ and observing that any sheet of spherical classes is necessarily mapped to a sheet of spherical classes by $\vartheta$. So, for $n=4$, the sheets $S, \theta(S)$ and $S^{\prime}$ are all isomorphic. However, for $n>4$, the sheets $S$ and $S^{\prime}$ have different dimension and are therefore not isomorphic as varieties. The intersection of any pair of distinct sheets is trivial, and the stratum is smooth if and only if the sheets it contains are so. It is enough to deal with $S$ and $S^{\prime}$. 
For the sheet $S$ we have $w_{S}=s_{\varepsilon_{1}+\varepsilon_{2}} s_{\varepsilon_{3}+\varepsilon_{4}} \cdots s_{\varepsilon_{n-1}+\varepsilon_{n}}=w_{0} w_{\Pi}$ for $\Pi=$ $\left\{\alpha_{1}, \alpha_{3}, \ldots, \alpha_{n-1}\right\}$. In this case $\theta\left(w_{S}\right) \neq w_{S}$. We choose the representative $\dot{w}_{S}=$ $\left(\begin{array}{ll}0 & L \\ L & 0\end{array}\right)$ where $L=\operatorname{diag}(J, J, \ldots, J)$ and $J=\left(\begin{array}{cc}0 & 1 \\ -1 & 0\end{array}\right)$.

Then, for $i=1, \ldots, h$ and $\epsilon_{i}= \pm 1, \dot{w}_{S}\left(Z\left(L_{\Pi}\right) \cap T^{w_{S}}\right) V_{S}$ is the disjoint union of the sets of matrices of the form $x(E, D)=\left(\begin{array}{cc}0 & E \\ E & D\end{array}\right)$ with $x_{i} \in k$ for $i=1, \ldots, h$ and

$$
E=\operatorname{diag}\left(E_{1}, \ldots, E_{h}\right), \quad E_{i}=\left(\begin{array}{cc}
0 & \epsilon_{i} \\
-\epsilon_{i} & 0
\end{array}\right), \quad D=\operatorname{diag}\left(-\epsilon_{1} x_{1} I_{2}, \ldots,-\epsilon_{h} x_{h} I_{2}\right) .
$$

Then $x(E, D)$ lies in $S$ only if there exists $\lambda \in k^{*} \operatorname{such}$ that $\operatorname{rk}(x(E, D)-\lambda I)=$ $n$. This is possible only if $D=\left(\lambda+\lambda^{-1}\right) I$. Conversely, if this is the case, a direct verification shows that $x(E, D)$ is either semisimple with eigenvalues $\lambda^{ \pm 1}$ or unipotent up to a sign. In addition, as $w_{\theta(S)} \neq w_{S}$ have the same length, and $w_{\theta(S)}$ is maximal among the elements $\tau \in W$ such that $\theta(S) \cap B \tau B \neq \emptyset$, we see that $\mathcal{O} \cap B w_{S} B=\emptyset$ for every $\mathcal{O} \subset \theta(S)$. Thus, any $x(E, D)$ satisfying $\operatorname{rk}(x(E, D)-\lambda I)=n$ lies in $S$. So, set theoretically, $S \cap \mathcal{S}_{w_{S}}$ is a disjoint union of $2^{h}$ affine lines. We conclude as in the previous cases.

For the sheet $S^{\prime}$ we have $w_{S^{\prime}}=s_{\beta} s_{1}=w_{0} w_{\Pi}$ for $\Pi=\left\{\alpha_{3}, \ldots, \alpha_{n}\right\}$ and $\beta=$

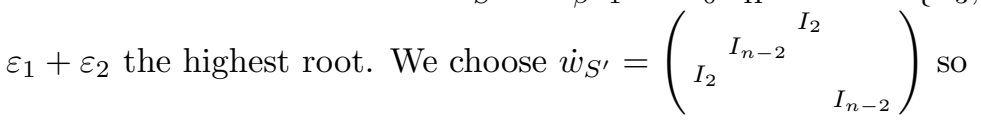

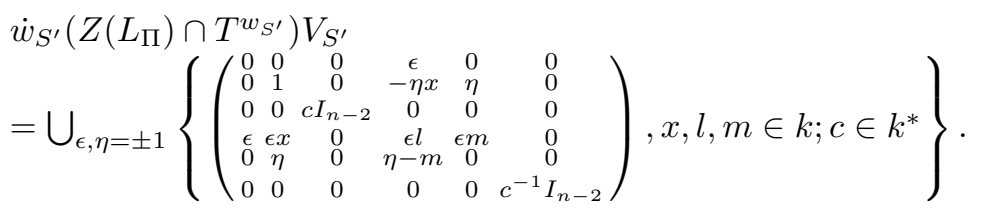

If $X \in \dot{w}_{S^{\prime}}\left(Z\left(L_{\Pi}\right) \cap T^{w_{S^{\prime}}}\right) V_{S^{\prime}}$ lies in $S^{\prime}$ then $r k(X-I)=2$. All elements satisfying this condition lie in $S^{\prime}$. Indeed, the centralizer of the representatives of the classes in $S^{\prime}$ in $O_{2 n}(k)$ is not contained in $S O_{2 n}(k)$ so elements that are $G L_{2 n}(k)$-conjugate, are also $S O_{2 n}(k)$-conjugate. Therefore, the argument used for the sheet $S^{\prime}$ in type $B_{n}$ applies. For such an $X$ we have

$$
c=1, \quad l=\eta x^{2}, \quad m=-\eta x .
$$

Hence the variety $S^{\prime} \cap \mathcal{S}_{w_{S^{\prime}}}$ is isomorphic to a disjoint union of 4 affine lines.

4.4.2. $D_{n}$ for $n$ odd. Let $n=2 h+1$. The only non-trivial sheets of spherical conjugacy classes are $R, \theta(R)$ and $S^{\prime}$ as follows:

$$
R=\bigcup_{\lambda \neq 0, \pm 1}\left(G \cdot\left(\begin{array}{ll}
\lambda I_{n} & \\
& \lambda^{-1} I_{n}
\end{array}\right)\right) \cup \pm \mathcal{O}_{\left(2^{n-1}, 1^{2}\right)}
$$

where $\mathcal{O}_{\left(2^{n-1}, 1^{2}\right)}$ is the unique unipotent conjugacy class corresponding to the partition $\left(2^{n-1}, 1^{2}\right)$; and $S^{\prime}$ is the same as for $n$ even and can be dealt with in the same way.

The sheet $S^{\prime}$ does not intersect $R$ nor $\theta(R)$. On the other hand, $R$ and $\theta(R)$ intersect in $\pm \mathcal{O}_{\left(2^{n-1}, 1^{2}\right)}$, hence the stratum containing them is not smooth.

Let us deal with $R$. The Weyl group element associated with it is $w_{R}=$ $s_{\varepsilon_{1}+\varepsilon_{2}} s_{\varepsilon_{3}+\varepsilon_{4}} \cdots s_{\varepsilon_{n-2}+\varepsilon_{n-1}}=w_{0} w_{\Pi}$ for $\Pi=\left\{\alpha_{1}, \alpha_{3}, \ldots, \alpha_{n-2}\right\}$. In this case $\theta\left(w_{R}\right)=w_{R}$. Let us consider the injective morphism $\iota: S O_{2 h}(k) \rightarrow S O_{n}(k)$ given

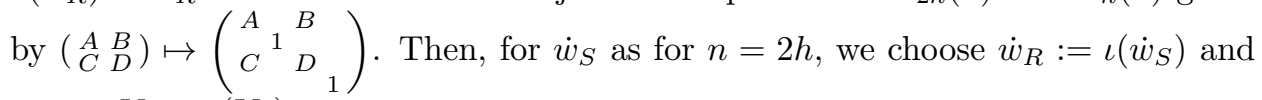
we get $V_{R}=\iota\left(V_{S}\right)$. 
Thus, for $i=1, \ldots, h$ and $\epsilon_{i}= \pm 1, \dot{w}_{R}\left(Z\left(L_{\Pi}\right) \cap T^{w_{R}}\right) V_{R}$ is the disjoint union

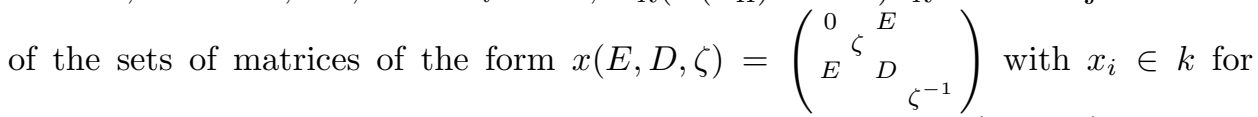
$i=1, \ldots, h, \zeta \in k^{*}$ and $E, D$ as for $n=2 h$. A matrix $x(E, D, \zeta)$ lies in $S$ only if there exists $\lambda \in k^{*}$ such that $\operatorname{rk}(x(E, D)-\lambda I) \leq n$. This is possible only if $D=\left(\lambda+\lambda^{-1}\right) I$ and $\zeta=\lambda^{ \pm 1}$. Conversely, if this is the case, a direct verification, making use of the computations for $n=2 h$ and the sheet $S$, shows that $x\left(E,\left(\lambda+\lambda^{-1}\right) I, \zeta\right)$ lies in $R$ if $\zeta=\lambda$ and it lies in $\theta(R)$ otherwise. Thus, set theoretically, $R \cap \mathcal{S}_{w_{R}}$ is a disjoint union of $2^{h}$ affine lines. We conclude as in the previous cases.

4.5. Exceptional groups. There are no non-trivial sheets of spherical conjugacy classes in types $E_{8}, F_{4}$, and $G_{2}$, so strata of spherical conjugacy classes consists of finitely many classes, hence they are smooth. Let us analyse the cases for $G$ simply-connected of type $E_{6}$ or $E_{7}$. For every $\beta \in \Phi$, we set $h_{\beta}: k^{*} \rightarrow T$ to be the co-character satisfying $\chi\left(h_{\beta}(\zeta)\right)=\zeta^{\frac{(\chi, \beta)}{(\beta, \beta)}}$ for every character $\chi$ of $T$. It is wellknown that every $t \in T$ has a unique expression as a product $t=\prod_{\alpha \in \Delta} h_{\alpha}\left(\zeta_{\alpha}\right)$, for $\zeta_{\alpha} \in k^{*}$. The simple roots in $E_{6}$ and $E_{7}$ will be enumerated as in [2].

4.5.1. The group of type $E_{6}$. Let $\omega \in k$ be a primitive fourth root of 1 and let $\zeta$ be a primitive third root of 1 . For $a \in k^{*}$, let

$$
p_{2, a}=h_{\alpha_{1}}\left(a^{2}\right) h_{\alpha_{2}}\left(a^{3}\right) h_{\alpha_{3}}\left(a^{4}\right) h_{\alpha_{4}}\left(a^{6}\right) h_{\alpha_{5}}\left(a^{5}\right) h_{\alpha_{6}}\left(a^{4}\right)
$$

and let $\mathcal{O}_{2 A_{1}}$ be the unipotent conjugacy class in $G$ with Bala-Carter label $2 A_{1}$. Then the only non-trivial sheet containing spherical classes is

$$
S=\left(\bigcup_{a \in k, a^{3} \neq 0,1} G \cdot p_{2, a}\right) \cup\left(\bigcup_{z \in Z(G)} z \mathcal{O}_{2 A_{1}}\right)
$$

By [5, Theorem 3.6], if $a \neq b$, then $p_{2, a}$ is not conjugate to $p_{2, b}$.

In this case, $w_{S}=w_{0} w_{\Pi}$ for $\Pi=\left\{\alpha_{3}, \alpha_{4}, \alpha_{5}\right\}$, so $w_{S}=s_{\beta} s_{\gamma}$ where $\beta=\alpha_{1}+$ $2 \alpha_{2}+2 \alpha_{3}+3 \alpha_{4}+2 \alpha_{5}+\alpha_{6}$ is the highest root and $\gamma=\alpha_{1}+\alpha_{3}+\alpha_{4}+\alpha_{5}+\alpha_{6}$ is the highest root in $\Phi \cap \beta^{\perp}=\Phi \cap \mathbb{Z}\left\{\alpha_{1}, \alpha_{3}, \alpha_{4}, \alpha_{5}, \alpha_{6}\right\}$.

We compute the set theoretical intersection $S \cap \mathcal{S}_{w_{S}}$ by detecting $\mathcal{O} \cap \mathcal{S}_{w_{S}}$ for each orbit in $S$.

Let us use a parametrization $x_{ \pm \alpha}(\xi)$ of the root subgroups $X_{ \pm \alpha}$, for $\alpha \in\{\beta, \gamma\}$ and $\xi \in k$, satisfying $x_{\alpha}(1) x_{-\alpha}(-1) x_{\alpha}(1)=n_{\alpha}$, with $n_{\alpha}$ commuting with the root subgroups associated with roots in $\pm \Pi$. We choose $\dot{w}_{S}:=n_{\beta} n_{\gamma}$.

We first consider $\mathcal{O}_{a}=G \cdot p_{2, a}$ for $a^{3} \neq 0,1$. Since $\beta\left(p_{2, a}\right)=\gamma\left(p_{2, a}\right)=a^{3}$ we have, for $\xi=\frac{1}{a^{-3}-1}$ :

$$
x_{-\beta}(\xi) x_{-\gamma}(\xi) p_{2, a} x_{-\gamma}(-\xi) x_{-\beta}(-\xi)=x_{-\beta}(-1) x_{-\gamma}(-1) p_{2, a}
$$

and

$$
\begin{aligned}
z_{a} & :=x_{\gamma}(1) x_{\beta}(1) x_{-\beta}(-1) x_{-\gamma}(-1) p_{2, a} x_{\beta}(-1) x_{\gamma}(-1) \\
& =\dot{w}_{S} p_{2, a} x_{\beta}\left(-a^{-3}-1\right) x_{\gamma}\left(-a^{-3}-1\right) \in w_{S} T U^{w_{S}} \cap \mathcal{O}_{a} .
\end{aligned}
$$

For $a \in k^{*}$ let $b, c \in k$ satify $b^{4}=c^{4}=a^{3}$. 
Conjugation of $z_{a}$ by $h_{\beta}(b) h_{\gamma}(c)$ gives

$$
\begin{aligned}
y_{a, b^{2}, c^{2}}:= & h_{\beta}(b) h_{\gamma}(c) z_{a} h_{\gamma}(c)^{-1} h_{\beta}(b)^{-1} \\
= & \dot{w}_{S} h_{\alpha_{1}}\left(a^{2}(b c)^{-2}\right) h_{\alpha_{3}}\left(a c^{-2}\right) h_{\alpha_{4}}\left(c^{2} b^{-2}\right) h_{\alpha_{5}}\left(a^{2} c^{-2}\right) h_{\alpha_{6}}\left(a b^{2} c^{-2}\right) \\
& \cdot x_{\beta}\left(-b^{2}\left(a^{-3}+1\right)\right) x_{\gamma}\left(-c^{2}\left(a^{-3}+1\right)\right) \in \mathcal{O}_{a} \cap \mathcal{S}_{w_{S}}
\end{aligned}
$$

which depends on $a, b^{2}, c^{2}$, for $c^{2}= \pm b^{2}$. Since $\mathcal{O}_{a} \cap \mathcal{S}_{w_{S}}$ is a single $\Gamma_{w_{S}}$-orbit and $\Gamma_{w_{S}}$ is generated by $h_{\beta}(\omega)$ and $h_{\gamma}(\omega)$, we have

$$
\left(\bigcup_{a^{3} \neq 0,1} \mathcal{O}_{a}\right) \cap \mathcal{S}_{w_{S}}=\bigcup_{\epsilon= \pm 1}\left(\bigcup_{a^{3} \neq 0,1 ; a^{3}=d^{2}} y_{a, d, \epsilon d}\right) \text {. }
$$

We now analyze the orbits in $Z(G) \mathcal{O}_{2 A_{1}}$. We recall that $Z(G)$ is generated by $p_{2, \zeta}$. A representative of $\mathcal{O}_{2 A_{1}}$ is $u=x_{-\beta}(-1) x_{-\gamma}(-1)$, so for $0 \leq l \leq 2$, the element

$$
y_{l}:=x_{\gamma}(1) x_{\beta}(1) p_{2, \zeta^{l}} u x_{\beta}(-1) x_{\gamma}(-1)=\dot{w}_{S} p_{2, \zeta^{l}} x_{\beta}(-2) x_{\gamma}(-2)
$$

lies in $p_{2, \zeta^{l}} \mathcal{O}_{2 A_{1}} \cap \mathcal{S}_{w_{S}}$. All other elements in this set are obtained by $\Gamma_{w_{S}}{ }^{-}$ conjugation:

$$
\begin{aligned}
y_{\zeta^{l}, i, j}:= & h_{\beta}\left(\omega^{i}\right) h_{\gamma}\left(\omega^{j}\right) y_{l} h_{\gamma}\left(\omega^{-j}\right) h_{\beta}\left(\omega^{-i}\right) \\
= & \dot{w}_{S} h_{\alpha_{1}}\left(\zeta^{-l}(-1)^{i+j}\right) h_{\alpha_{3}}\left(\zeta^{l}(-1)^{j}\right) h_{\alpha_{4}}\left((-1)^{i+j}\right) h_{\alpha_{5}}\left((-1)^{j} \zeta^{-l}\right) h_{\alpha_{6}}\left((-1)^{i+j} \zeta^{l}\right) \\
& \cdot x_{\beta}\left(-2(-1)^{i}\right) x_{\gamma}\left(-2(-1)^{j}\right)
\end{aligned}
$$

hence

$$
\left(\bigcup_{z \in Z(G)} z \mathcal{O}_{2 A_{1}}\right) \cap \mathcal{S}_{w_{S}}=\bigcup_{\epsilon= \pm 1}\left(\bigcup_{a^{3}=1 ; 1=d^{2}} y_{a, d, \epsilon d}\right) .
$$

By Remark 4.3, $S \cap \mathcal{S}_{w}$ is the union of two disjoint irreducible components, each isomorphic to the image of the curve $x^{3}=y^{2}$, for $x, y \neq 0$, through the morphism $(x, y) \mapsto\left(x^{-1}, x y^{-1}, x^{2} y^{-1}, x, y\left(x^{-3}+1\right)\right)$.

4.5.2. The group of type $E_{7}$. For $a \in k^{*}$, let

$$
q_{3, a}=h_{\alpha_{1}}\left(a^{2}\right) h_{\alpha_{2}}\left(a^{3}\right) h_{\alpha_{3}}\left(a^{4}\right) h_{\alpha_{4}}\left(a^{6}\right) h_{\alpha_{5}}\left(a^{5}\right) h_{\alpha_{6}}\left(a^{4}\right) h_{\alpha_{7}}\left(a^{3}\right)
$$

and let $\omega$ be a fourth primitive root of 1 . Let $\mathcal{O}_{3 A_{1}^{\prime \prime}}$ be the unipotent conjugacy class in $G$ with Bala-Carter label $3 A_{1}^{\prime \prime}$. Then

$$
S=\left(\bigcup_{a \in k, a \neq 0, \pm 1} G \cdot q_{3, a}\right) \cup\left(\bigcup_{z \in Z(G)} z \mathcal{O}_{3 A_{1}^{\prime \prime}}\right)
$$

is the only non-trivial sheet containing spherical classes. Here, $w_{S}=w_{0} w_{\Pi}$ for $\Pi=\left\{\alpha_{2}, \alpha_{3}, \alpha_{4}, \alpha_{5}\right\}$ so $w_{S}=s_{\beta} s_{\gamma} s_{\alpha_{7}}$ for $\beta=2 \alpha_{1}+2 \alpha_{2}+3 \alpha_{3}+4 \alpha_{4}+3 \alpha_{5}+2 \alpha_{6}+\alpha_{7}$ the highest root, and $\gamma=\alpha_{2}+\alpha_{3}+2 \alpha_{4}+2 \alpha_{5}+2 \alpha_{6}+\alpha_{7}$ the highest root in $\Phi \cap \beta^{\perp}$.

Let us use a parametrization $x_{ \pm \alpha}(\xi)$ of the root subgroups $X_{ \pm \alpha}$, for $\alpha \in\left\{\beta, \gamma, \alpha_{7}\right\}$ and $\xi \in k$, satisfying $x_{\alpha}(1) x_{-\alpha}(-1) x_{\alpha}(1)=n_{\alpha}$, with $n_{\alpha}$ commuting with root subgroups associated with roots in $\pm \Pi$. We choose $\dot{w}_{S}:=n_{\beta} n_{\gamma} n_{\alpha_{7}}$. We will argue as we did for $E_{6}$. 
Let us first consider $\mathcal{O}_{a}=G \cdot q_{3, a}$ for $a^{2} \neq 0,1$. Since $\beta\left(q_{3, a}\right)=\gamma\left(q_{3, a}\right)=$ $\alpha_{7}\left(q_{3, a}\right)=a^{2}$ we have, for $\xi=\frac{1}{a^{-2}-1}$ :

$$
\begin{aligned}
& x_{-\beta}(\xi) x_{-\gamma}(\xi) x_{-\alpha_{7}}(\xi) q_{3, a} x_{-\alpha_{7}}(-\xi) x_{-\gamma}(-\xi) x_{-\beta}(-\xi) \\
& =x_{-\beta}(-1) x_{-\gamma}(-1) x_{-\alpha_{7}}(-1) q_{3, a}
\end{aligned}
$$

and

$$
\begin{aligned}
z_{a} & :=x_{\gamma}(1) x_{\beta}(1) x_{\alpha_{7}}(1) x_{-\beta}(-1) x_{-\gamma}(-1) x_{-\alpha_{7}}(-1) q_{3, a} x_{\alpha_{7}}(-1) x_{\beta}(-1) x_{\gamma}(-1) \\
& =\dot{w}_{S} q_{3, a} x_{\beta}\left(-a^{-2}-1\right) x_{\gamma}\left(-a^{-2}-1\right) x_{\alpha_{7}}\left(-a^{-2}-1\right) \in w_{S} T U^{w_{S}} \cap \mathcal{O}_{a} .
\end{aligned}
$$

For $a \in k^{*}$, let $b, c, d \in k$ satisfy $b^{4}=c^{4}=d^{4}=a^{2}$.

Conjugation of $z_{a}$ by $h_{\beta}(b) h_{\gamma}(c) h_{\alpha_{7}}(d)$ gives

$$
\begin{aligned}
y_{a, b^{2}, c^{2}, d^{2}}:= & h_{\beta}(b) h_{\gamma}(c) h_{\alpha_{7}}(d) z_{a} h_{\alpha_{7}}(d)^{-1} h_{\gamma}(c)^{-1} h_{\beta}(b)^{-1} \\
= & \dot{w}_{S} h_{\alpha_{2}}\left(a c^{-2}\right) h_{\alpha_{3}}\left(a^{2} b^{-2} c^{-2}\right) h_{\alpha_{5}}\left(a b^{-2}\right) h_{\alpha_{7}}\left(a^{3} b^{-2} c^{-2} d^{-2}\right) \\
& \cdot x_{\beta}\left(-b^{2}\left(a^{-2}+1\right)\right) x_{\gamma}\left(-c^{2}\left(a^{-2}+1\right)\right) x_{\alpha_{7}}\left(-d^{2}\left(a^{-2}+1\right)\right) \in \mathcal{O}_{a} \cap \mathcal{S}_{w_{S}}
\end{aligned}
$$

which depends on $a, b^{2}, c^{2}, d^{2}$, for $c^{2}= \pm b^{2}= \pm d^{2}= \pm a$. As all elements in $w_{S} T^{w_{S}} U^{w_{S}} \cap \mathcal{O}_{a}$ form a single orbit for the group $\Gamma_{w_{S}}$, which is generated by $h_{\beta}(\omega), h_{\gamma}(\omega)$ and $h_{\alpha_{7}}(\omega)$, we have

$$
\left(\bigcup_{a^{2} \neq 0,1} \mathcal{O}_{a}\right) \cap \mathcal{S}_{w_{S}}=\bigcup_{\epsilon, \eta, \theta= \pm 1}\left(\bigcup_{a^{2} \neq 0,1} y_{a, \epsilon a, \eta a, \theta a}\right)
$$

and, for $b^{2}=\epsilon a, c^{2}=\eta a, d^{2}=\theta a$ we have

$$
\begin{aligned}
y_{a, \epsilon a, \eta a, \theta a}= & \dot{w}_{S} h_{\alpha_{2}}(\eta) h_{\alpha_{3}}(\epsilon \eta) h_{\alpha_{5}}(\epsilon) h_{\alpha_{7}}(\epsilon \eta \theta) \\
& \cdot x_{\beta}\left(-\epsilon\left(a^{-1}+a\right)\right) x_{\gamma}\left(-\eta\left(a^{-1}+a\right)\right) x_{\alpha_{7}}\left(-\theta\left(a^{-1}+a\right)\right) .
\end{aligned}
$$

Let us now consider the orbits in $Z(G) \mathcal{O}_{3 A_{1}^{\prime \prime}}$. We recall that $Z(G)$ is generated by $q_{3,-1}$. The class $\mathcal{O}_{3 A_{1}}$ is represented by $u=x_{-\beta}(-1) x_{-\gamma}(-1) x_{-\alpha_{7}}(-1)$, so, for $\xi= \pm 1$, the element

$$
\begin{aligned}
y_{\xi} & :=x_{\gamma}(1) x_{\beta}(1) x_{\alpha_{7}}(1) q_{3, \xi} u x_{\alpha_{7}}(-1) x_{\beta}(-1) x_{\gamma}(-1) \\
& =\dot{w}_{S} q_{3, \xi} x_{\beta}(-2) x_{\gamma}(-2) x_{\alpha_{7}}(-2) \in q_{3, \xi} \mathcal{O}_{3 A_{1}^{\prime \prime}} \cap \mathcal{S}_{w_{S}} .
\end{aligned}
$$

All other elements in this set are obtained by $\Gamma_{w_{S}}$-conjugation:

$$
\begin{aligned}
& h_{\beta}\left(\omega^{i}\right) h_{\gamma}\left(\omega^{j}\right) h_{\alpha_{7}}\left(\omega^{l}\right) y_{\xi} h_{\alpha_{7}}\left(\omega^{-l}\right) h_{\gamma}\left(\omega^{-j}\right) h_{\beta}\left(\omega^{-i}\right) \\
& =\dot{w}_{S} h_{\alpha_{2}}\left(\xi(-1)^{j}\right) h_{\alpha_{3}}\left((-1)^{i+j}\right) h_{\alpha_{5}}\left(\xi(-1)^{i}\right) h_{\alpha_{7}}\left(\xi(-1)^{i+j+l}\right) \\
& \quad \cdot x_{\beta}\left(-2(-1)^{i}\right) x_{\gamma}\left(-2(-1)^{j}\right) x_{\alpha_{7}}\left(-2(-1)^{l}\right)
\end{aligned}
$$

We conclude that

$$
S \cap \mathcal{S}_{w_{S}}=\bigcup_{\epsilon, \eta, \theta= \pm 1}\left(\bigcup_{a^{2} \neq 0} y_{a, \epsilon a, \eta a, \theta a}\right),
$$

which, by Remark 4.3 is isomorphic to a disjoint union of 8 copies of an affine line, with coordinate ring $k\left[a+a^{-1}\right]$. 


\section{REFERENCES}

[1] M. Bulois, Sheets of symmetric Lie algebras and Slodowy slices, J. Lie Theory 21 (2011), no. 1, 1-54. MR2797818 (2012f:14090)

[2] N. Bourbaki, Éléments de mathématique (French), Masson, Paris, 1981. Groupes et algèbres de Lie. Chapitres 4, 5 et 6. [Lie groups and Lie algebras. Chapters 4, 5 and 6]. MR647314 (83g:17001)

[3] N. Cantarini, G. Carnovale, and M. Costantini, Spherical orbits and representations of $\mathcal{U}_{\epsilon}(\mathfrak{g})$, Transform. Groups 10 (2005), no. 1, 29-62, DOI 10.1007/s00031-005-1002-z. MR2127340 (2005m:17020)

[4] G. Carnovale, Spherical conjugacy classes and involutions in the Weyl group, Math. Z. 260 (2008), no. 1, 1-23, DOI 10.1007/s00209-007-0244-y. MR2413339 (2009d:20091)

[5] G. Carnovale, A classification of spherical conjugacy classes in good characteristic, Pacific J. Math. 245 (2010), no. 1, 25-45, DOI 10.2140/pjm.2010.245.25. MR2602680 (2011c:20095)

[6] G. Carnovale, Lusztig's partition and sheets (with an appendix by M. Bulois), Math. Res. Lett. 22 (2015), no. 3, 645-664, DOI 10.4310/MRL.2015.v22.n3.a1. MR3350097

[7] G. Carnovale and M. Costantini, On Lusztig's map for spherical unipotent conjugacy classes, Bull. Lond. Math. Soc. 45 (2013), no. 6, 1163-1170, DOI 10.1112/blms/bdt048. MR3138484

[8] G. Carnovale and F. Esposito, On sheets of conjugacy classes in good characteristic, Int. Math. Res. Not. IMRN 4 (2012), 810-828. MR2889158

[9] K. Y. Chan, J.-H. Lu, and S. K.-M. To, On intersections of conjugacy classes and Bruhat cells, Transform. Groups 15 (2010), no. 2, 243-260, DOI 10.1007/s00031-010-9084-7. MR2657442 (2011e:20068)

[10] M. Costantini, A classification of unipotent spherical conjugacy classes in bad characteristic, Trans. Amer. Math. Soc. 364 (2012), no. 4, 1997-2019, DOI 10.1090/S0002-9947-2011-053900. MR2869197 (2012m:20076)

[11] M. Costantini, A classification of spherical conjugacy classes, arXiv:1503.03827.

[12] M. Goto and F. D. Grosshans, Semisimple Lie algebras, Marcel Dekker, Inc., New York-Basel, 1978. Lecture Notes in Pure and Applied Mathematics, Vol. 38. MR0573070 (58 \#28084)

[13] A. Grothendieck, Séminaire de Géométrie Algébrique du Bois Marie - 1960-61 - Revêtements étales et groupe fondamental - (SGA 1), Lecture Notes in Mathematics 224, Berlin; New York: Springer-Verlag (1971).

[14] R. Hartshorne, Algebraic geometry, Graduate Texts in Mathematics, No. 52, Springer-Verlag, New York-Heidelberg, 1977. MR0463157 (57 \#3116)

[15] X. He and G. Lusztig, A generalization of Steinberg's cross section, J. Amer. Math. Soc. 25 (2012), no. 3, 739-757, DOI 10.1090/S0894-0347-2012-00728-0. MR2904572

[16] A. E. Im Hof, The sheets in a classical Lie algebra, Ph.D. thesis, Basel, http:// edoc.unibas.ch/257/ (2005).

[17] P. I. Katsylo, Sections of sheets in a reductive algebraic Lie algebra (Russian), Izv. Akad. Nauk SSSR Ser. Mat. 46 (1982), no. 3, 477-486, 670. MR661143 (84k:17005)

[18] I. Losev, 1-dimensional representations and parabolic induction for W-algebras, Adv. Math. 226 (2011), no. 6, 4841-4883, DOI 10.1016/j.aim.2010.12.021. MR2775887 (2012i:17009)

[19] J.-H. Lu, On a dimension formula for spherical twisted conjugacy classes in semisimple algebraic groups, Math. Z. 269 (2011), no. 3-4, 1181-1188, DOI 10.1007/s00209-010-0776-4. MR2860282(2012m:20077)

[20] G. Lusztig, On conjugacy classes in a reductive group, arxiv:1305.7168, to appear in the Proceedings of Vogan60 Conference.

[21] G. Lusztig and N. Spaltenstein, Induced unipotent classes, J. London Math. Soc. (2) 19 (1979), no. 1, 41-52, DOI 10.1112/jlms/s2-19.1.41. MR527733 (82g:20070)

[22] D. Mumford, Geometric invariant theory, Ergebnisse der Mathematik und ihrer Grenzgebiete, Neue Folge, Band 34, Springer-Verlag, Berlin-New York, 1965. MR0214602 (35 \#5451)

[23] A. Premet, Commutative quotients of finite $W$-algebras, Adv. Math. 225 (2010), no. 1, 269306, DOI 10.1016/j.aim.2010.02.020. MR2669353 (2011j:17024)

[24] A. Premet and L. Topley, Derived subalgebras of centralisers and finite $W$-algebras, Compos. Math. 150 (2014), no. 9, 1485-1548, DOI 10.1112/S0010437X13007823. MR3260140

[25] A. Sevostyanov, Algebraic group analogues of the Slodowy slices and deformations of Poisson $W$-algebras, Int. Math. Res. Not. IMRN 8 (2011), 1880-1925, DOI 10.1093/imrn/rnq139. MR2806525 (2012f:20141) 
[26] A. Sevostyanov, A proof of De Concini-Kac-Procesi conjecture II. Strictly transversal slices to conjugacy classes in algebraic groups, arXiv:1403.4108.

Dipartimento di Matematica, Torre Archimede - via Trieste 63 - 35121 Padova, Italy E-mail address: carnoval@math.unipd.it

Dipartimento di Matematica, Torre Archimede - via Trieste 63 - 35121 Padova, Italy E-mail address: esposito@math.unipd.it 